\title{
Brief
}

\section{Prosthetic graft remnant-related pseudoaneurysm after left ventricular assist device explantation: A case report}

\author{
Gregory N. Messner, D0, Chayanin Vatcharasiritham, MD, Igor Gregoric, MD, Branislav Radovancevic, MD, \\ Peggy Odegaard, RN, Scott D. Flamm, MD, and O. H. Frazier, MD, Houston, Tex
}

From the Cardiovascular Surgical Research
Laboratories, Texas Heart Institute at St
Luke's Episcopal Hospital, Houston, Tex.

Received for publication Nov 13, 2002; accepted for publication Dec 16, 2002.

Address for reprints: O. H. Frazier, MD, Cardiovascular Surgical Research Laboratories, Texas Heart Institute at St Luke's Episcopal Hospital, PO Box 20345, Houston, TX 77225-0345 (E-mail: knowlin@ heart.thi.tmc.edu)

J Thorac Cardiovasc Surg 2004;127:259-61

$0022-5223 / \$ 30.00$

Copyright ( $\odot 2004$ by The American Association for Thoracic Surgery

doi:10.1016/S0022-5223(03)00129-6
$\mathrm{P}$

seudoaneurysm of the ascending aorta is a rare but severe complication of cardiac surgery. Most cases occur after coronary artery bypass grafting (CABG) and aortic valvular procedures, and many others occur after cardiac transplantation. Thus far, the only reported case of a pseudoaneurysm related to a left ventricular assist device (LVAD) is a pseudoaneurysm related to the prosthetic graft itself. ${ }^{1}$ We report here the first case of a pseudoaneurysm related to LVAD explantation.

\section{Clinical Summary}

In December 1999, a 47-year-old woman with a history of progressive dyspnea on exertion presented with a syncopal episode. She was admitted to a local hospital, where congestive heart failure was diagnosed. Echocardiography revealed an ejection fraction of $10 \%$. The patient was transferred to St Luke's Episcopal Hospital for further evaluation and treatment. There she underwent placement of an intra-aortic balloon pump, followed by simultaneous implantation of an LVAD (Thoratec VAD; Thoratec Corporation, Pleasanton, Calif) and performance of the Batista procedure. Three months later, in February 2000, the patient's condition had improved to the point that the LVAD could be removed. Immediately after its removal, the patient experienced postoperative bleeding that required mediastinal exploration to control. The patient's sternum was closed 1 day later. The patient did well for almost 1 year, until she complained of a painful swelling of the upper sternum. A computed tomography (CT) scan of the chest showed fluid anterior and posterior to the sternum extending back to the margin of the ascending thoracic aorta, a finding compatible with an abscess. The abscess was incised and drained after achievement of local anesthesia. A culture grown from a sample of the fluid grew methicillin-resistant Staphylococcus aureus. Intravenous vancomycin was instituted for 1 month. On follow-up examination, a repeat CT scan showed a 2.3-cm-diameter saccular aneurysm involving the distal ascending thoracic aorta and a parasternal collection of fluid containing air suggestive of an abscess. The abscess was surgically drained, and intravenous vancomycin was continued at a dosage of $0.5 \mathrm{~g} / \mathrm{d}$. Again, a culture grown from a sample of the drained fluid grew methicillin-resistant $S$ aureus.

Magnetic resonance angiography (MRA) was performed to confirm the diagnosis of aneurysm of the ascending aorta. This revealed a pseudoaneurysm of the ascending aorta measuring $2.0 \times 2.4 \times 3.0 \mathrm{~cm}$ (Figure 1). Consequently, the patient was scheduled for resection of the pseudoaneurysm. The femoral vessels were exposed and readied for cannulation, and the sternum was opened with an oscillating saw. Because the pseudoaneurysm was adherent to the sternum, the opening of the sternum caused inadvertent entry into the pseudoaneurysm. The femoral vessels were immediately cannulated, and femorofemoral bypass was commenced. The patient was cooled to $18^{\circ} \mathrm{C}$, and circulatory arrest combined with retrograde cerebral perfusion was initiated. Then the sternum was slowly opened. After reduction of the blood flow to $500 \mathrm{~mL} / \mathrm{min}$, the patient was placed in the Trendelenburg position to prevent air embolism. The pseudoaneurysm, together with the remnant of the prosthetic graft still attached to the ascending aorta, was resected. The pre-existing anastomosis between the prosthetic graft and the ascending aorta was found to be disrupted for about one third of its entire circumference. The disrupted portion of the anastomosis was 


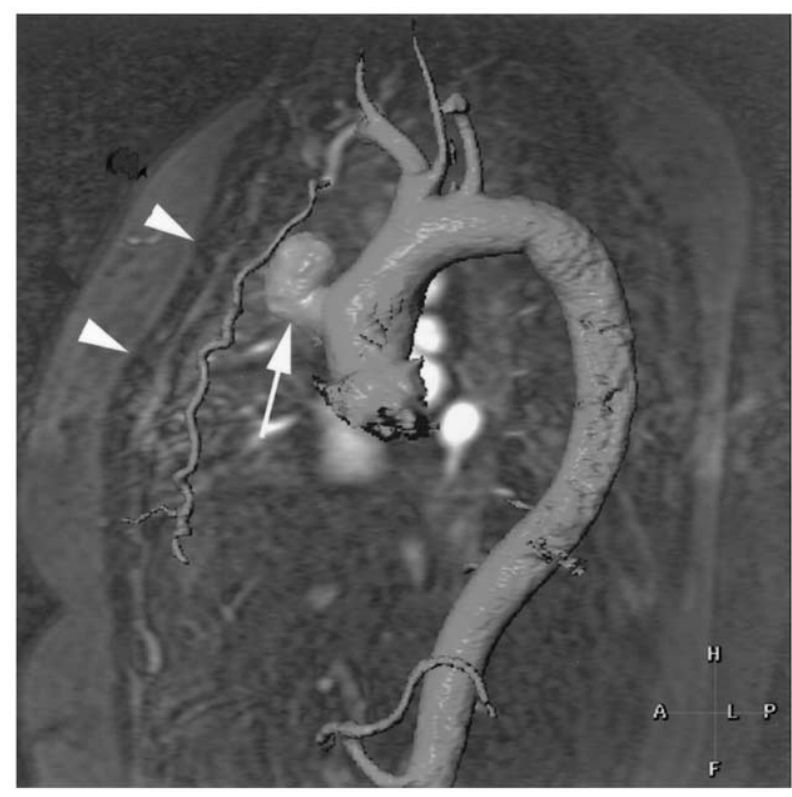

Figure 1. Surface-shaded rendering of the thoracic aorta in the lateral projection superimposed on a single sagittal slice of the chest, as acquired by means of 3-dimensional MRA. The arrowheads denote the sternum and show the proximity of the pseudoaneurysm (arrow) when considering re-exploration. The twisting line between the pseudoaneurysm and the sternum indicates the right internal thoracic artery.

resected, leaving a $3 \times 2-\mathrm{cm}$ defect at the aorta, and further dissection of the ascending aorta was done to facilitate crossclamping. After crossclamping, the patient was warmed to $32^{\circ} \mathrm{C}$ and then subjected to retrograde cerebral perfusion. The defect at the aorta was repaired by using an oval Dacron patch. The crossclamp was then released, and the patient was warmed to $37^{\circ} \mathrm{C}$ and weaned off cardiopulmonary bypass. The sternum was left open until the next day, when the patient was brought back to the surgical theater for placement of an omental graft and closure of the sternum. Even though cultures of the resected pseudoaneurysm specimen did not grow any organisms, an antibiotic therapy regimen was instituted. Postoperatively, the patient remained stable and was discharged home after 2 weeks.

At 1 year of follow-up, the patient was stable with no neurologic deficits. A CT scan of the chest showed normal findings and confirmed that the Dacron patch repair was still intact (Figure 2). The antibiotic regimen was discontinued on the recommendation of an infectious disease consultant.

\section{Discussion}

This report documents the first case of a pseudoaneurysm related to the removal of an LVAD. CT scanning and MRA confirmed the diagnosis, and surgical intervention was performed to correct the problem.

Although cardiac transplantation is the therapeutic option of choice and currently the most frequent treatment for end-stage heart failure, wider use of this approach has been limited by several issues. These include fewer available donor hearts than

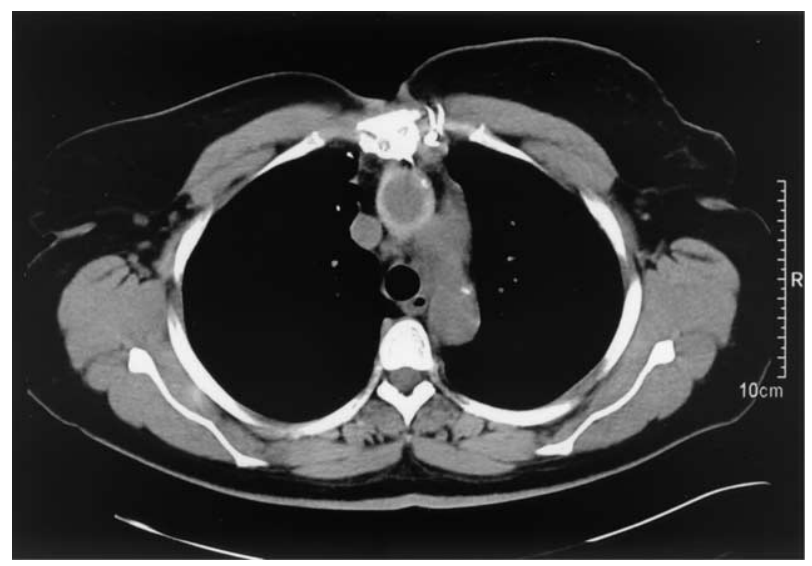

Figure 2. CT scan of the chest, taken at 1 year of follow-up, showing normal findings and confirming that the Dacron repair graft was still intact.

transplant candidates and the resultant long waiting period for an organ. In addition, there are the usual problems of rejection faced by all transplant recipients and the heart-specific problem of coronary graft atherosclerosis, which can significantly limit the longterm survival of heart recipients. ${ }^{2}$ During the past 2 decades, temporary mechanical circulatory support has become a widely accepted option for patients with intractable heart failure, particularly those awaiting cardiac transplantation. An unexpected benefit of prolonged LVAD use has been improved ventricular function in patients with idiopathic and dilated cardiomyopathies. In a few cases in which LVAD removal was necessitated by device failure or device-related infection, the patient's native cardiac function had recovered sufficiently to avoid subsequent transplantation or reimplantation of an assist device. ${ }^{3}$ Prolonged mechanical unloading of the failing heart by an LVAD promotes ventricular recovery (ie, improved cardiothoracic ratio, end-diastolic dimension, ejection fraction, pulmonary capillary wedge pressure, and pulmonary vascular resistance and also striking histologic improvement of the myocardium). ${ }^{4,5}$ This unexpected benefit of prolonged LVAD use has led to another indication for LVAD support: bridging to recovery. Although this new use of an LVAD is promising, this case demonstrates that the device might also lead to unexpected complications, such as the development of a pseudoaneurysm.

Typically, pseudoaneurysm of the ascending aorta is thought to be an unusual and potentially fatal complication of cardiovascular surgery. Most pseudoaneurysms are associated with aortic valve or CABG surgery and are often infectious in origin. In several reported series, infected pseudoaneurysms occurred along the aortic suture lines after cardiac transplantation. However, to our knowledge, there has never been any report of a case of an infected pseudoaneurysm of the prosthetic graft remnant that remains attached to the ascending aorta after LVAD removal. The only similar report in the literature is of a pseudoaneurysm of the outflow graft itself in a patient supported with a Novacor N100 left ventricular assist system (Baxter Healthcare Corp, Oakland, Calif). ${ }^{1}$ 
Because the ascending aorta has been the preferred site for arterial cannulation for primary heart operations for the past 2 decades, and because the number of CABG and aortic valvular procedures involving entry into the ascending aorta has increased, pseudoaneurysms of the thoracic aorta have become more frequent. ${ }^{6,7}$ Predisposing factors include graft infection, dissected native aorta, and possibly tissue necrosis after excessive use of biologic glue. ${ }^{8}$ Pseudoaneurysms are the result of disruption of at least one layer of the vessel wall and are contained by the remaining vascular layers or the surrounding structures of the mediastinum with or without development of a neointima. In this respect, pseudoaneurysms differ from true aneurysms, in which all 3 vascular layers remain intact. ${ }^{9}$ Although this pseudoaneurysm was the result of an infection and breakdown of the anastomotic graft stump, we were able to repair the defect with a prosthetic patch, with no untoward effects. ${ }^{10}$

As the present case makes clear, follow-up is essential for LVAD-supported patients who undergo device removal and are left with a remnant of the prosthetic graft attached to the ascending aorta because the remnant has the potential to become a focus of infection or to develop into a pseudoaneurysm. In cases of mediastinal infection in this subgroup of patients, early MRA should be done because it might easily reveal the presence of a pseudoaneurysm. Surgical management is essential in the setting of pseudoaneurysms after LVAD explantation.

\section{References}

1. Knosalla C, Weng Y, Buz S, Loebe M, Hetzer R. Pseudoaneurysm of the outflow graft in a patient with Novacor N100 LVAS system. Ann Thorac Surg. 2000;70:1594-6.

2. Frazier OH. Mechanical cardiac assistance: historical perspectives. Semin Thorac Cardiovasc Surg. 2000;12:207-20.

3. Frazier OH. Future direction of cardiac assistance. Semin Thorac Cardiovasc Surg. 2000;12:251-8.

4. Frazier OH, Benedict CR, Radovancevic B, Bick RJ, Capek P, Springer WE, et al. Improved left ventricular function after chronic left ventricular unloading. Ann Thorac Surg. 1996;62:675-82.

5. Hetzer R, Muller JH, Weng Y, Meyer R, Dandel M. Bridging-torecovery. Ann Thorac Surg. 2001;71:S109-13.

6. Sullivan KL, Steiner RM, Smullens SN, Griska L, Meister SG. Pseudoaneurysm of the ascending aorta following cardiac surgery. Chest. 1988;92:138-43.

7. Razzouk A, Gundry S, Wang N, Heyner R, Sciolaro C, Van Arsdell G, et al. Pseudoaneurysms of the aorta after cardiac surgery of chest trauma. Am Surg. 1993;59:818-23.

8. Katsumata T, Moorjani N, Vaccari G, Westaby S. Mediastinal false aneurysm after thoracic aortic surgery. Ann Thorac Surg. 2000;70:54752.

9. Marx M, Gardiner GA, Miller RH. The truth about false aneurysm. Am J Radiol. 1985;145:193-4.

10. Jacobs MJ, Reul GJ, Gregoric I, Cooley DA. In-situ replacement and extra-anatomic bypass for the treatment of infected abdominal grafts. Eur J Vasc Surg. 1991;5:83-6. 